operational definition and Core Outcome Set for wellbeing in doctors is needed to meaningfully progress the work in this field. Method. The Centre for Workforce Wellbeing (C4WW), a collaboration between the University of Southampton and Health Education England, was created to support research into the nature, assessment and enhancement of wellbeing in physicians. A Systematic Review of wellbeing measures used in doctors and the robustness of those measures, along with surveys of $250 \mathrm{UK}$ doctors of all grades and specialities and patient and public involvement is informing what a core outcome set could be. A Delphi Study among 37 UK experts has been initiated to establish the consensus Core Outcome Set.

Result. Publication of research into doctors' wellbeing is growing internationally. In the UK alone data are being captured by multiple national organisations including: the Care Quality Commission, General Medical Council, British Medical Association and the Royal Colleges. Health and Social Care Organisations are, therefore, keen to "do something" and are spending money on wellbeing interventions with little, or no, evidence base and a lack of appropriate, comparable evaluation. A Core Outcome Set for measuring wellbeing in doctors is ethically required to reduce waste, to replace burnout measures and to refine wellbeing interventions.

Conclusion. Wellbeing measures that actually measure wellbeing, and not burnout, which are validated, reliable and practical, are needed to inform local organisational, national government and international research policy. An absence of burnout does not equate to wellbeing. The focus of measurement needs to shift to capture in what contexts we thrive, not just survive. If everyone used the same Core Outcome Set to measure mental wellbeing, direct comparisons could be made, and money invested, in creating infrastructure, processes and cultures that really work.

Health Education England funded PhD.

\section{What is mental wellbeing?}

\section{David Baldwin ${ }^{1 \star}$, Julia Sinclair ${ }^{1}$ and Gemma Simons ${ }^{2}$}

${ }^{1}$ Professor of Psychiatry and Head of Mental Health Group, University of Southampton Faculty of Medicine Honorary Professor of Psychiatry, University of Cape Town, South Africa Visiting Professor, Shandong Mental Health Centre, China and ${ }^{2}$ Centre for Workforce Wellbeing, University of southampton

${ }^{*}$ Corresponding author.

\section{doi: 10.1192/bjo.2021.631}

Aims. To explore the theory of wellbeing and to propose an operational definition for wellbeing in doctors.

Hypothesis: An operational definition for wellbeing in doctors is needed in order for it to be measured and interventions to improve it developed.

Background. There is no internationally recognised definition for wellbeing and yet wellbeing is an increasingly fashionable topic of research and development, including in doctors. This is because wellbeing can be described using either hedonist, or eudonist philosophy and there is a lack of conceptual clarity about what wellbeing is, and how it works. Research into the measurement of mental wellbeing has been dominated by individualist societies, with the inherent bias towards measuring self-centred components and not the other-orientated components that might be valued more in collectivist societies and by doctors.

Method. The Centre for Workforce Wellbeing (C4WW), a collaboration between the University of Southampton and Health Education England, was created to support research into the nature, assessment and enhancement of wellbeing in physicians. A literature review of the philosophy, definition and measurement of wellbeing was undertaken with a focus on mental wellbeing at work and specifically in doctors.

Result. A concept map of the relationship between wellbeing terms has been created and was used to understand and classify where mental wellbeing itself was being defined and measured in studies, as opposed to a component of wellbeing, or determinant of wellbeing. Thematic analysis was used to develop an operational definition of wellbeing for doctors.

Conclusion. Measurement of wellbeing and interventions for wellbeing cannot be developed if you cannot clearly define what wellbeing is. An operational definition of mental wellbeing in doctors is ethically required to prevent research waste and to allow us to identify and recreate when doctors thrive, not just survive.

Health Education England funded $\mathrm{PhD}$.

\section{A state hospital survey of movement disorders including intention tremor}

\author{
Nigel Bark ${ }^{1 *}$, Sung-Ai Kim² and George Eapen ${ }^{3}$ \\ ${ }^{1}$ Albert Einstein College of Medicine, Bronx Psychiatric Center; \\ ${ }^{2}$ Bronx Psychiatric Center (Retired) and ${ }^{3}$ Rainbow Babies and \\ Childrens Hospital \\ ${ }^{*}$ Corresponding author.
}

doi: 10.1192/bjo.2021.633

Aims. In a survey of movement disorders in patients in a State Hospital the finger-nose test was included because of increasing interest in the cerebellum in schizophrenia. It was expected that this would reflect the pathobiology of schizophrenia and be unrelated to the type of medication.

Background. Abnormalities of movement and involuntary movements have gone from being considered part of schizophrenia to side-effects of medication to now demonstrably present in those who have never taken anti-psychotic medication. Soft neurological signs (SNS) are increased in schizophrenia, unrelated to medication, considered not to indicate brain localization, yet often include the finger-nose test which localizes to the cerebellum.

Method. All available patients in a State Hospital were examined for movement disorders. They were rated on the following scales: Abnormal Involuntary Movement Scale (AIMS) for Tardive Dyskinesia (TD), Simpson-Angus Neurological Rating Scale for Parkinsonism (SANRS), Barnes Akathisia Scale (BAS), a Dystonia scale and the finger-nose test.

Result. 250 patients were included, 174 were examined or observed for movement disorder: 120 had no missing data, 54 refused part of the exam. Their mean age was $47,62 \%$ male, $53 \%$ black, 26\% Hispanic, 17\% white.

Medication: First Generation Antipsychotic (FGA) 35 (mean CPZ equivalent dose:1177mg), Second Generation Antipsychotic (SGA) 159 (734mg), both FGA and SGA 56 (1907mg), no antipsychotic 3; anticholinergic or amantidine: FGA 57\%, SGA $16 \%$, both FGA and SGA: $50 \%$.

Tardive Dyskinesia: all 23\%, FGA 36\%, SGA 25\%, both 7\%

Parkinsonism: all 38\%, FGA 43\%, SGA 33\%, both 34\%

Akathisia: all 3\%, FGA 0\%, SGA 4\%, both 3\%

Pseudo-akathisia: FGA $11 \%$, SGA $4 \%$, both $13 \%$

Dystonia: all $10 \%$, FGA 13\%, SGA $11 \%$, both $8 \%$

Intention Tremor: all 16\%, FGA 0\%, SGA $21 \%$, both $16 \%$

Half of those with Intention Tremor had Parkinsonism, a third had TD and a half were on anti-Parkinson medication.

None of these differences were statistically significant at $\mathrm{p}=0.05$ though intention tremor did show a trend $(p=0.08)$. The difference between FGA and SGA only became significant when all movement disorders were added together with those on anticholinergics with no movement disorder. 